\title{
Bayesian Networks of Customer Satisfaction Survey Data
}

\author{
Silvia Salini* \\ University of Milan, Italy
}

Ron S. Kenett

KPA Ltd., Raanana, Israel and University of Torino, Torino, Italy

\begin{abstract}
A Bayesian Network is a probabilistic graphical model that represents a set of variables and their probabilistic dependencies. Formally, Bayesian Networks are directed acyclic graphs whose nodes represent variables, and whose arcs encode the conditional dependencies between the variables. Nodes can represent any kind of variable, be it a measured parameter, a latent variable or a hypothesis. They are not restricted to representing random variables, which forms the "Bayesian" aspect of a Bayesian network. Efficient algorithms exist that perform inference and learning in Bayesian Networks. Bayesian Networks that model sequences of variables are called Dynamic Bayesian Networks. Harel et. al (2007) provide a comparison between Markov Chains and Bayesian Networks in the analysis of web usability from e-commerce data. A comparison of regression models, SEMs, and Bayesian networks is presented Anderson et. al (2004). In this paper we apply Bayesian Networks to the analysis of Customer Satisfaction Surveys and demonstrate the potential of the approach. Bayesian Networks offer advantages in implementing models of cause and effect over other statistical techniques designed primarily for testing hypotheses. Other advantages include the ability to conduct probabilistic inference for prediction and diagnostic purposes with an output that can be intuitively understood by managers.
\end{abstract}

Keywords: Bayesian Networks, Customer Satisfaction, Eurobarometer, Service Quality

* Address for correspondence: Silvia Salini, Department of Economics, Business and Statistics. University of Milan. Via Conservatorio 7. 20122 Milan Italy.

e-mail: silvia.salini@unimi.it 


\section{Introduction}

A Bayesian Network is a probabilistic graphical model that represents a set of variables and their probabilistic dependencies. Formally, Bayesian Networks are directed acyclic graphs whose nodes represent variables, and whose arcs encode the conditional dependencies between the variables. Nodes can represent any kind of variable, be it a measured parameter, a latent variable or a hypothesis. They are not restricted to representing random variables, which forms the "Bayesian" aspect of a Bayesian network. Efficient algorithms exist that perform inference and learning in Bayesian Networks. We begin with some theoretical background on Bayesian Networks $(\mathrm{BN})$ and proceed with two applications of $\mathrm{BN}$ to customer satisfaction survey data analysis. The first example is from a survey of customers of a complex electronic product combining software, electronics and mechanical features. The product requires support at different levels and the survey is assessing satisfaction levels of customers from different features of the product and related services. The second example is from the Eurobarometer public opinion surveys conducted on behalf of the DirectorateGeneral for Education and Culture of the European Commission each Spring and Autumn. We focus on four services: fixed telephone, electricity supply, gas supply, water supply and, for each service, examine three aspects: accessibility, price and quality. we conclude with a discussion and some direction for further research.

\section{Theoretical Background: Bayesian Network}

Bayesian networks, also known as Belief Networks belong to the family of probabilistic Graphical Models (GM). These graphical structures are used to represent knowledge about an uncertain domain. In particular, each node in the graph represents a random variable, while the edges between the nodes represent probabilistic dependencies among the corresponding 
random variables. These conditional dependencies in the graph are often estimated by using known statistical and computational methods. Hence, Bayesian networks combine principles from graph theory, probability theory, computer science and statistics.

Bayesian networks $(\mathrm{BN})$ correspond to another GM structure known as a directed acyclic graph (DAG) that is popular in the Statistics, the Machine Learning and the Artificial Intelligence societies. $\mathrm{BN}$ are both mathematically rigorous and intuitively understandable. They enable an effective representation and computation of the joint probability distribution over a set of random variables (Pearl, 2000).

The structure of a directed acyclic graph is defined by two sets: the set of nodes (vertices) and the set of directed edges. The nodes represent random variables and are drawn as circles labelled by the variables names. The edges represent direct dependence among the variables and are drawn by arrows between nodes. In particular, an edge from node $X_{i}$ to node $X_{j}$ represents a statistical dependence between the corresponding variables. Thus, the arrow indicates that a value taken by variable $X_{j}$ depends on the value taken by variable $X_{i}$, or roughly speaking that variable $X_{i}$ 'influences' $X_{j}$. Node $X_{i}$ is then referred to as a 'parent' of $X_{j}$ and, similarly, $X_{j}$ is referred to as the 'child' of $X_{i}$. An extension of these genealogical terms is often used to define the sets of 'descendents' - the set of nodes that can be reached on a direct path from the node, or 'ancestors' nodes - the set of nodes from which the node can be reached on a direct path. The structure of the acyclic graph guarantees that there is no node that can be its own ancestor or its own descendent. Such a condition is of vital importance to the factorization of the joint probability of a collection of nodes as seen below. Note that although the arrows represent direct causal connection between the variables, the reasoning process can operate on $\mathrm{BN}$ by propagating information in any direction.

A Bayesian network reflects a simple conditional independence statement. Namely that each variable is independent of its non-descendents in the graph given the state of its parents. This 
property is used to reduce, sometimes significantly, the number of parameters that are required to characterize the joint probability distribution (JPD) of the variables. This reduction provides an efficient way to compute the posterior probabilities given the evidence (Lauritzen et al, 1988, Pearl, 2000, Jensen, 2001).

In addition to the DAG structure, which is often considered as the "qualitative" part of the model, one needs to specify the "quantitative" parameters of the model. The parameters are described in a manner which is consistent with a Markovian property, where the conditional probability distribution (CPD) at each node depends only on its parents. For discrete random variables, this conditional probability is often represented by a table, listing the local probability that a child node takes on each of the feasible values - for each combination of values of its parents. The joint distribution of a collection of variables can be determined uniquely by these local conditional probability tables (CPT).

Formally, a Bayesian network $B$ is an annotated acyclic graph that represents a joint probability distribution over a set of random variables $\mathbf{V}$. The network is defined by a pair $B=\langle G, \Theta\rangle$, where $G$ is the directed acyclic graph whose nodes $X_{1}, X_{2}, \ldots, X_{n}$ represents random variables, and whose edges represent the direct dependencies between these variables. The graph $G$ encodes independence assumptions, by which each variable $X_{i}$ is independent of its non-descendents given its parents in $G$. The second component $\Theta$ denotes the set of parameters of the network. This set contains the parameter $\theta_{x_{i} \mid \pi_{i}}=P_{B}\left(x_{i} \mid \pi_{i}\right)$ for each realization $x_{i}$ of $X_{i}$ conditioned on $\pi_{i}$, the set of parents of $X_{i}$ in $G$. Accordingly, $B$ defines a unique joint probability distribution over $\mathbf{V}$, namely:

$$
P_{B}\left(X_{1}, X_{2}, \ldots, X_{n}\right)=\prod_{i=1}^{n} P_{B}\left(X_{i} \mid \pi_{i}\right)=\prod_{i=1}^{n} \theta_{X_{i} \mid \pi_{i}}
$$

For simplicity of representation we omit the subscript $B$ henceforth. If $X_{\mathrm{i}}$ has no parents, its local probability distribution is said to be unconditional, otherwise it is 
conditional. If the variable represented by a node is observed, then the node is said to be an evidence node, otherwise the node is said to be hidden or latent.

The complexity of a domain may be reduced by models and algorithms that describe an approximated reality. When variable interactions are too intricate for application of an analytic model, we may represent current knowledge about the problem, such as a cause generating at least one effect (Pearl, 2000), where the final effect is the target of the analysis; for example in Figure 1 the network topology (Lauritzen and Spiegelhalter, 1988) of cause and effect is built by choosing a set of variables (i.e. "Visit Africa", "Smoking") that describe the domain (a patient presents some problems and the physician wants to identify his/her disease and the correct therapy).

The domain knowledge allows experts to draw an arc to a variable from each of its direct causes (i.e. visiting Africa may cause tuberculosis).

Given a BN that specified the JPD in a factored form, one can evaluate all possible inference queries by marginalization, i.e., summing out over 'irrelevant' variables. Two types of inference support are often considered: predictive support for node $X_{i}$, based on evidence nodes connected to $X_{i}$ through its parent nodes (called also top-down reasoning), and diagnostic support for node $X_{i}$, based on evidence nodes connected to $X_{i}$ through its children nodes (called also bottom-up reasoning). In general, the full summation (or integration) over discrete (continuous) variables is called exact inference and known to be an NP-hard problem. Some efficient algorithms exist to solve the exact inference problem in restricted classes of networks. In many practical settings the $\mathrm{BN}$ is unknown and one needs to learn it from the data. This problem is known as the BN learning problem, which can be stated informally as follows: Given training data and prior information (e.g., expert knowledge, causal relationships), estimate the graph topology (network structure) and the parameters of the JPD in the BN. 
Learning the $\mathrm{BN}$ structure is considered a harder problem than learning the $\mathrm{BN}$ parameters. Moreover, another obstacle arises in situations of partial observablity when nodes are hidden or when data is missing. In the simplest case of known BN structure and full observability, the goal of learning is to find the values of the $\mathrm{BN}$ parameters (in each $\mathrm{CPD}$ ) that maximize the (log)likelihood of the training dataset. This dataset contains $m$ cases that are often assumed to be independent. Given training dataset $\boldsymbol{\Sigma}=\left\{\mathbf{x}, \ldots, \mathbf{x}_{\mathbf{m}}\right\}$, where $\boldsymbol{x}_{\boldsymbol{l}}=\left(x_{l 1}, \ldots, x_{l n}\right)^{T}$, and the parameter set $\boldsymbol{\Theta}=\left(\boldsymbol{\theta}, \ldots, \boldsymbol{\theta}_{\mathbf{n}}\right)$, where $\boldsymbol{\theta}_{i}$ is the vector of parameters for the conditional distribution of variable $X_{i}$ (represented by one node in the graph), the log-likelihood of the training dataset is a sum of terms, one for each node:

$$
\log L(\Theta \mid \Sigma)=\sum_{m} \sum_{n} \log P\left(x_{l i} \mid \pi_{i}, \theta_{i}\right)
$$

The log-likelihood scoring function decomposes according to the graph structure, hence, one can maximize the contribution to the log-likelihood of each node independently. Another alternative is to assign a prior probability density function to each parameter vector and use the training data to compute the posterior parameter distribution and the Bayes estimates. To compensate for zero-occurrences of some sequences in the training dataset, one can use appropriate (mixtures of) conjugate prior distributions, e.g., the Dirichlet prior for the multinomial case as in the above backache example or the Wishart prior for the Gaussian case. Such an approach results in a maximum a-posteriori estimate and known also as the equivalent sample size (ESS) method.

Figure 1: An example of a causal network (Lauritzen and Spiegelhalter, 1988).

Bayesian Networks are gaining popularity within a wide range of application areas such as risk management (Cornalba et al, 2007), web data analysis (Harel et al, 2008) and management science in general (Rugerri et al, 2007, Kenett et al, 2008). Availability of 
software for analyzing Bayesian Networks is further expanding their role in decision analysis and decision support systems (Jensen, 2001).

\section{Application to Customer Satisfaction Data Analysis}

\subsection{The Annual Customer Satisfaction Survey}

In this Section a BN is implemented in order to analyze customer satisfaction survey data from a complex electronic product combining software, electronics, and mechanical features, The product requires support at different levels. The survey is assessing satisfaction levels of customers from different features of the product and related services. An important issue in product customer satisfaction surveys is to find which aspects of the product and services influence overall satisfaction, the recommendation levels and the repurchasing intentions. BN is a useful tool to analyze such impact with the advantage that it produces graphical output that are easy to understand and make it simple to conduct probabilistic inference for prediction.

In order to show these advantages, a $\mathrm{BN}$ has been applied to data collected from 266 companies (customers) participating in an Annual Customer Satisfaction Survey. The data refers to a questionnaire composed of 81 questions. The dataset and the questionnaire are available on http://www.economia.unimi.it/projects/CSProject/.

For each customer we have several demographic variables such as: Country, Segmentation, Age of equipment and Profitability.

A basic frequency analysis shows that the majority of customers come from Germany, do not belong to a specific industry sector and have a Break-Even Profitability; while age of equipment and customer seniority have an heterogeneous distribution.

The first part of the questionnaire evaluates Overall Satisfaction, with a score going from 1 (very low satisfaction) to 5 (very high satisfaction) and two specific variables (questions): 1) 
repurchasing intention and recommendation level measured with a score going from 1 (very unlikely) to 5 (very likely). We observe that $64.9 \%$ are very likely to repurchase products.

In the second part of the questionnaire, there is a set of questions (items) grouped according to different dimensions: Equipment, Sales Support, Technical Support, Training, Supplies and Media,, Customer Portal, Administrative Support, Terms-Conditions and Prices and Site Planning and Installation. For each question we have two types of scores: the item evaluation score, going from 1 (Strongly disagree) to 5 (Strongly agree), and the item importance level (low $=1$, medium $=2$, high $=3$, and Not Applicable). For each dimension, there is an overall evaluation. The dimension with the highest level of satisfaction is Training and the one with the lowest level is Terms-Condition and Prices. We consider the overall evaluation for each dimensions.

The data is analyzed with a basic BN presented in Figure 2. The network combines background information with a learned network generated using the Greedy Thick Thinning algorithm implemented in the GeNIe version 2.0 software (http://genie.sis.pitt.edu).

Figure 2: Bayesian Network from Customer Satisfaction Survey data

Note that the dimensions that influence Overall Satisfaction, Recommendation level and Repurchasing intention are Suppliers, Equipment and Technical Support. The other dimensions appear independent to each other. Moreover Overall Satisfaction, Recommendation and Repurchasing are influenced by the country of the customer. A bar plot representation of the nodes of the BN is shown in Figure 3.

Figure 3: Bar chart of Bayesian Network on Customer Satisfaction Survey data 
On the basis of the network we can perform various diagnostic checks. Figure 4 presents distributions of various variables conditioned on the Recommendation being at it's highest level.

Figure 4: Diagnostic distributions conditioned on the Recommendation being at its highest level.

It is important to observe that Overall Satisfaction and Repurchasing change in the same direction than Recommendation. Note also that the percentages of Technical Support are 7\%, $13 \%, 16 \%, 38 \%$ and $26 \%$ in Figure 3 and $5 \%, 8 \%, 9 \%, 42 \%$ and $37 \%$ in Figure 4 , so high level of Recommendation correspond to high satisfaction level from Technical Support. This information has a practical relevance, an intervention to improve the Technical support may increase Repurchase, Recommendation and Overall Satisfaction.

\subsection{Service Quality: Europeans Opinion about Utilities}

In this Section we use BNs to analyse the opinion of Europeans about Utilities that emerges from Eurobarometer Survey. The satisfaction of citizen depends on the country macro variables, on the year considered, on the privatization level of the service, on the social and demographical characteristics of the consumer (Fiorio et. al 2007). Moreover various aspects of service can be evaluated in different way and can influenced each other. The same services do not seem to be independent between them, in fact they have been proposals for indices of total satisfaction that combine the services (Ferrari and Salini, 2007). In this complex situation, characterized by a high number of variable, with nonlinear relationships and combined dependencies, a $\mathrm{BN}$ is an appropriate model. Again, BNs provide a causal explanation using observable variables within a single multivariate model and analysis of nonlinear relationships contained in ordinal measurements. 
In the next subsection we present the Eurobarometer Survey data. In the second part we construct a $\mathrm{BN}$ analysis and comment on the results.

\subsubsection{Eurobarometer Survey Data}

Eurobarometer public opinion surveys (henceforth, EB) have been conducted on behalf of the Directorate General for Education and Culture of the European Commission each Spring and Autumn, since the Autumn of 1973. They have included Greece, since the Autumn of 1980, Portugal and Spain, since the Autumn of 1985, the former German Democratic Republic, since the Autumn of 1990 and Austria, Finland and Sweden, from the Spring of 1995 onwards.

An identical set of questions is asked to a representative sample of the population over fifteen years old in each Member State. In each household, the respondent is drawn at random. All interviews are face-to-face in people's homes and in the appropriate national language. A detailed analysis of the Eurobarometer data can be found on the official Eurobarometer Web site. ${ }^{1}$ The questions concern various aspects, including support and benefit for EU membership, support for an EU constitution, satisfaction with EU democracy and the single currency, general outlook on life and so on.

The regular sample in standard Eurobarometer surveys is 1000 people per country except Luxembourg (600) and the United Kingdom (1000 in Great Britain and 300 in Northern Ireland). In order to monitor the integration of the five new Länder into unified Germany and the European Union, 2000 persons have been sampled in Germany since the Eurobarometer 34: 1000 in East Germany and 1000 in West Germany.

In each of the 15 Member States, the survey is carried out by national institutes associated with the "INRA (Europe) European Coordination Office". This network of institutes was

\footnotetext{
${ }^{1} \mathrm{http}: / /$ europa.eu.int/comm/public_opinion/
} 
selected by tender. All institutes are members of the "European Society for Opinion and Marketing Research" (ESOMAR) and comply with its standards.

Each survey comes with a set of weights obtained, using marginal and intercellular weighting, carried out on the basis of the population description provided by EUROSTAT in the Regional Statistics Yearbook (data for 1997 or 1996).

In the years 2000, 2002 and 2004 the Eurobarometer surveys included some questions relating to Services of General Interest (henceforth, SGI). The SGI considered are mobile telephone services, fixed telephone services, electricity supply services, gas supply services, water supply services, postal services, transport services within towns/cities and rail services between towns/cities. The criteria used to analyse these services are accessibility, the price of the services, the quality of the services, the clarity of the information aimed at EU Consumers, how fair the terms and conditions of the contracts applied to the services are, Consumer complaints and how they are handled and Customer Service.

In this application we consider four Services: fixed telephone, electricity supply, gas supply, water supply and, for each service, examine three aspects: accessibility, price and quality. The data from three years $(2000,2002,2004)$ is pooled, and the analysis is carried out on the entire data set in order to obtain a comparison between countries and years. Hence the final data set is structured in the following way: the rows (near 47.000) represent the respondents belonging to different Countries (near 15.000 for each year in each question), the columns refer to items (12 dimensions): the accessibility of the fixed telephone service (SGIaccT), the accessibility of the electricity supply service (SGIaccE), the accessibility of the gas supply service (SGIaccG), the accessibility of the water supply (SGIaccW), the price of the fixed telephone service (SGIpriT), the price of the electricity supply service (SGIpriE), the price of the gas supply service (SGIpriG), the price of the water supply (SGIaccW), the quality of the fixed telephone service (SGIquaT), the quality of the electricity supply service (SGIquaE), the 
quality of the gas supply service (SGIquaG), the quality of the water supply (SGIquaW). We have three levels for accessibility (not accessible, difficult to access, easy to access), three levels for price (excessive, unfair, fair) and four levels for quality (very bad, fairly bad, fairly good, very good).

\subsubsection{Bayesian Network on Eurobarometer Data}

The data was analyzed using a basic $\mathrm{BN}$ presented in Figure 5 The network combines background information with a learned network generated using the Greedy Thick Thinning algorithm implemented in the GeNIe version 2.0 software.

Figure 5: Bayesian Network on Eurobarometer Survey data

It is important to notice that "country" seems to influence only electricity supply service, which affects all the other services. "Year" influences electricity supply service and fixed telephone service. Relating to the aspects of service, accessibility is connected to quality and price and quality is connected to price for all services. Moreover, it is evident that services are not independent from each other. Looking as an example to the price of fixed telephone service (SGIpriT), it also depends on access, quality and price of all the other services. A bar plot representation of the node of BN is shown in Figure 6.

Figure 6: Bar plot BN on Eurobarometer Survey data

One the basis of the network, as in the previous case, we can perform various diagnostic checks. Figures 7 and 8 present distributions of various variables conditioned on the Country, Italy and Ireland respectively. 
Figure 7: Evidence for Italy

Figure 8: Evidence for Ireland

Generally, the opinion in Ireland is better than in Italy. For example the percentage of "Fair" for the price of gas service is $75 \%$ in Ireland versus 52\% for Italy. Also for the price of fixed telephone, $66 \%$ "Fair" in Ireland and $46 \%$ in Italy. For the quality of water, it happens that only the $19 \%$ of Italian think that it is "very good" versus $62 \%$ in Ireland. Figure 9 presents distributions of price of fixed telephone conditioned on the quality and access of fixed telephone being at their highest level.

Figure 9: Diagnostic distributions of price of fixed telephone service conditioned on the access of fixed telephone service and quality of fixed telephone service being at their highest level.

Good quality and easy access positively influence the opinion about price, in fact the distribution of price of fixed telephone service change from 58\% of Fair, 31\% of Unfair and $11 \%$ of Excessive (see Figure 6) to $73 \%$ of Fair, $21 \%$ of Unfair and $7 \%$ of Excessive. This is importance evidence for suppliers and legislators. Figure 10 presents distributions of price of fixed telephone conditioned on the price of the other services being at its highest level.

Unfair prices on the other services make to get worse the judgment on prices also of the fixed telephone. Probably the dissatisfaction generates a hostile climate and greater expectations. With $100 \%$ of prices considered "unfair" in the other services, the distribution of 
price considerations of fixed telephone will be $19 \%$ as Fair, $76 \%$ of Unfair and $5 \%$ of Excessive.

Figure 10: Diagnostic distributions of price of fixed telephone service conditioned on the price of electricity supply service, price of water supply service and price of gas supply service being at their lowest level

\section{Conclusions}

Bayesian Networks have been rarely used so far in analyzing customer satisfaction data. In this paper we demonstrate the advantages of the approach and, hopefully, raise awareness to such possibilities. Other techniques include Rasch models, Decision Trees, Structural Equation Models, Neural Networks and Analytical Hierarchical Processes. Hopefully this paper will trigger further research and applications of such techniques in analyzing data from customer satisfaction surveys.

\section{Acknowledgements}

The Eurobarameter data for this work has been provided by the 6th Framework Programme project Understanding Privatisation Policy: Political Economy and Welfare Effects of the European Union. The ABC customer satisfaction survey data is from a benchmarking project initiated in 2005 by the authors and several other partners from the University of Torino under the Diego de Castro foundation, to promote the application of advanced statistical techniques to the analysis of customer satisfaction data. for more on the project look at http://www.economia.unimi.it/projects/CSProject\%20/. 


\section{References}

Cornalba, C., Kenett, R. and Giudici, P. (2007) "Sensitivity Analysis of Bayesian Networks with Stochastic Emulators", ENBIS-DEINDE proceedings, University of Torino, Turin, Italy.

Cowell, R.G., Dawid, A. P., Lauritzen S.L., \& Spiegelhalter D.J. (1999). Probabilistic Networks and Expert Systems. New York, USA: Springer.

Ferrari P.A. and Salini S. (2007), Measuring Service Quality: the opinion of Europeans about Utilities, FEEM working papers, Milan, Italy.

Fiorio C.V., Florio M., Salini S., Ferrari P.A. (2007), Consumers' Attitudes on Services of General Interest in the EU: Accessibility, Price and Quality 2000-2004 - In: Servizi Pubblici: Nuove Tendenze nella Regolamentazione, nella Produzione e nel Finanziamento ; [a cura di] M. Marrelli, F. Padovano, I. Rizzo. - [s.l.] : Franco Angeli.

Harel, A. Kenett, R. and Ruggeri, F. (2008) "Modeling Web Usability Diagnostics on the basis of Usage Statistics" in Statistical Methods in eCommerce Research, W. Jank and G. Shmueli editors, Wiley.

Heckerman, D., (1995). A tutorial on learning with Bayesian networks. Microsoft Research tech. report MSR-TR-95-06. Revised November 1996, from http://research.microsoft.com.

Jensen, F.V., (2001). Bayesian networks and decision graphs. New York, USA: Springer.

Kenett, R.S. (2006). "On the Planning and Design of Sample Surveys", Journal of Applied Statistics Vol. 33, No. 4, 405-415.

Kenett, R., De Frenne, A., Tort-Martorell, X. and McCollin, C. (2008), The Statistical Efficiency Conjecture, to appear in Applying Statistical Methods in Business and Industrythe state of the art, Coleman, S., Greenfield, T., and Montgomery, D. (editors), Wiley. 
Lauritzen, S.L. (1995). The EM algorithm for graphical association models with missing data. Computational Statistics and Data Analysis 19, 191-201.

Lauritzen, S. L., Spiegelhalter, D. J. (1988), "Local computations with probabilities on graphical structures and their application to expert systems". Journal of the Royal Statistical Society, Series B (Methodological), 50(2):157-224.

Murphy K.P. (2001). An introduction to graphical models. A Brief Introduction to Graphical Models and Bayesian Networks. May 10, from http://www.cs.ubc.ca.

Pearl, J. 91995) Causal diagrams for empirical research, Biometrika, 1995, pp. 669-710.

Pearl, J. (2000) Causality: Models, Reasoning, and Inference, Cambridge University Press

Rugerri, F., Kenett, R. and Faltin F. (2007). The Encyclopaedia of Statistics in Quality and Reliability, Wiley. 


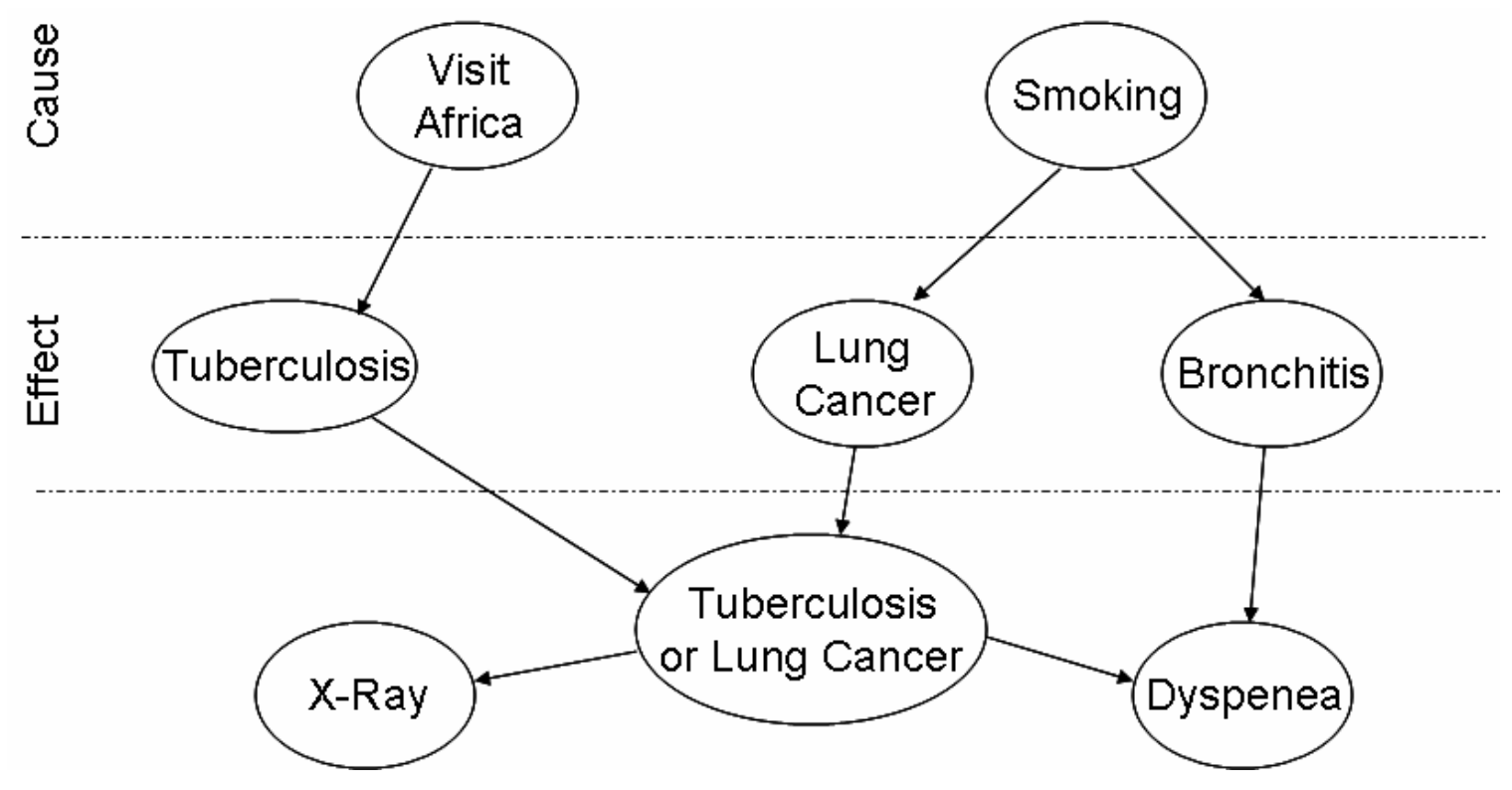

Figure 1: An example of a causal network (Lauritzen and Spiegelhalter, 1988).

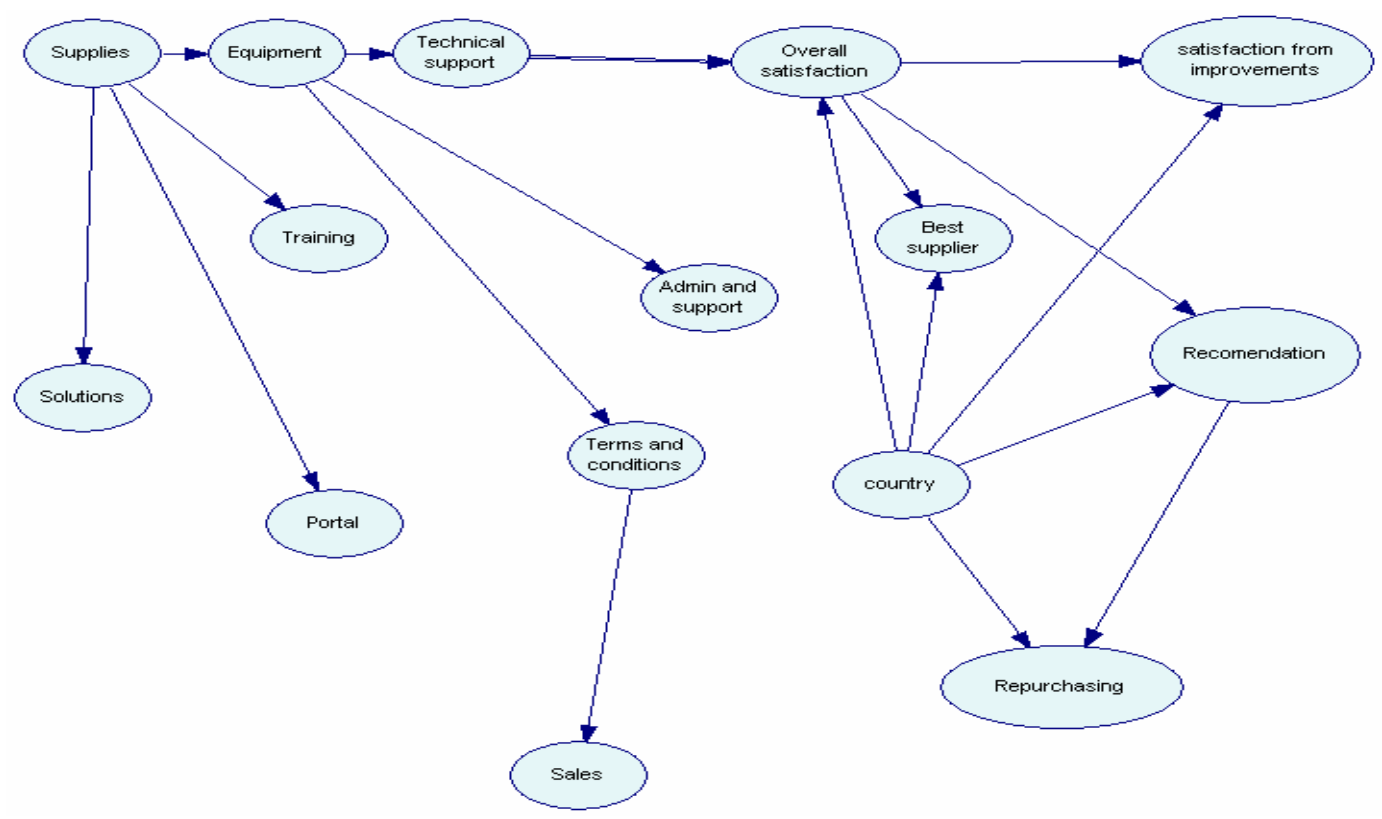


Figure 2: Bayesian Network from Customer Satisfaction Survey data

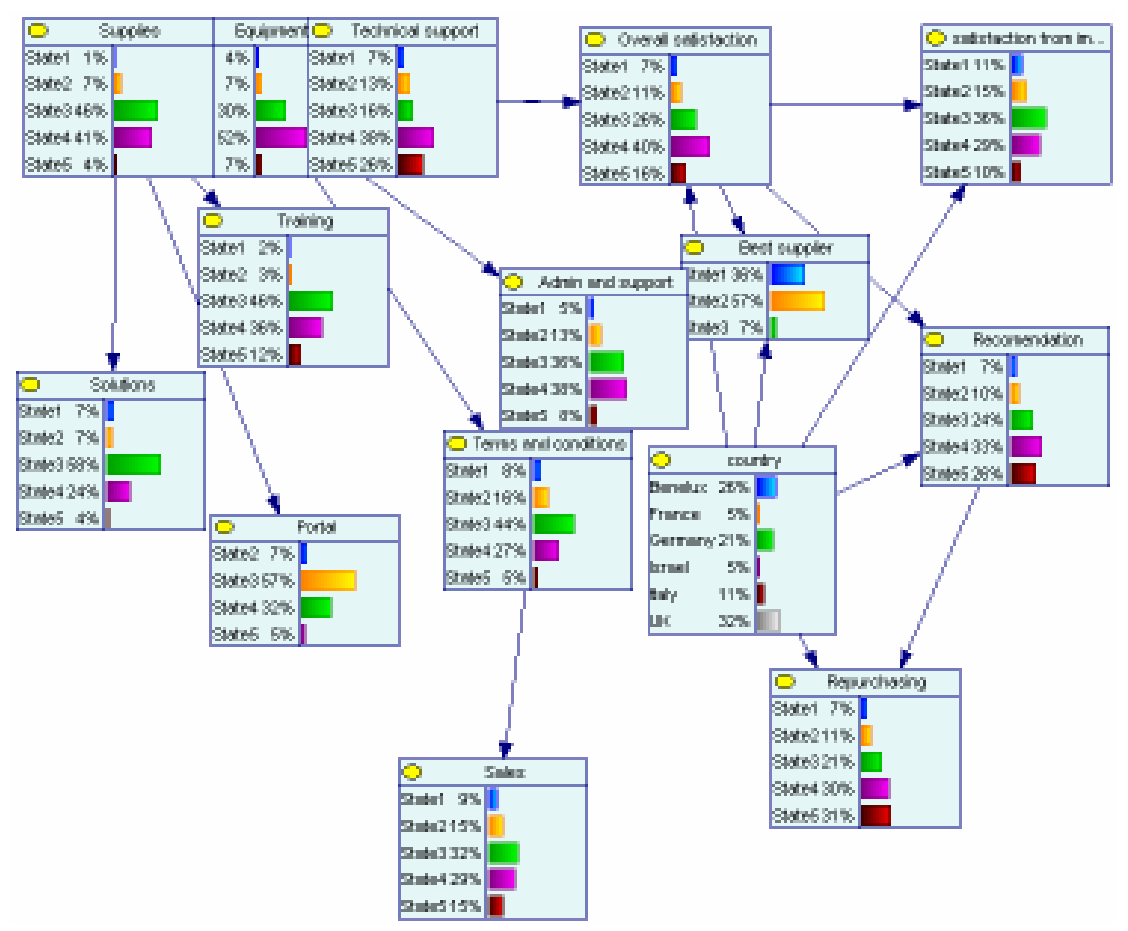

Figure 3: Bar chart of Bayesian Network on Customer Satisfaction Survey data 


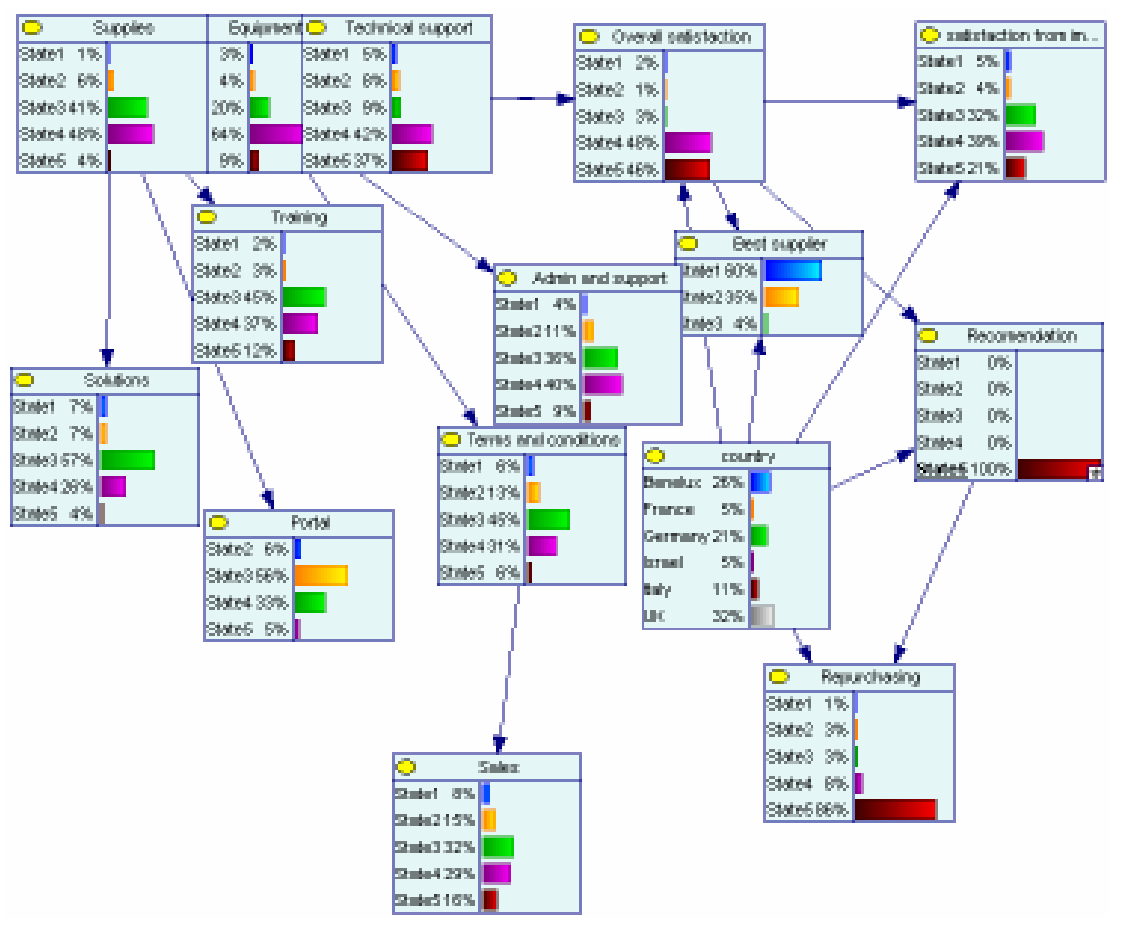

Figure 4: Diagnostic distributions conditioned on the Recommendation being at it's highest level.

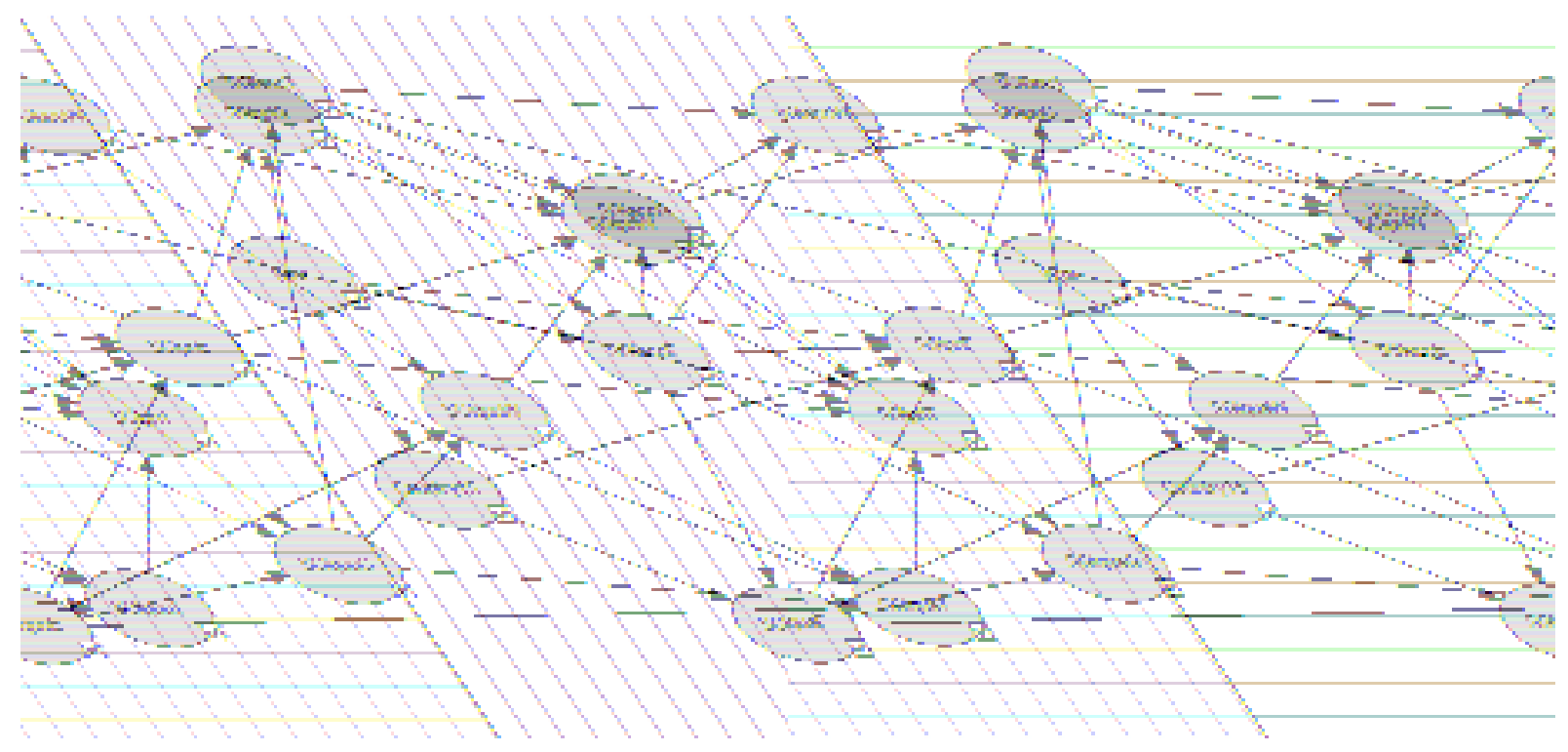

Figure 5: Bayesian Network on Eurobarometer Survey data 


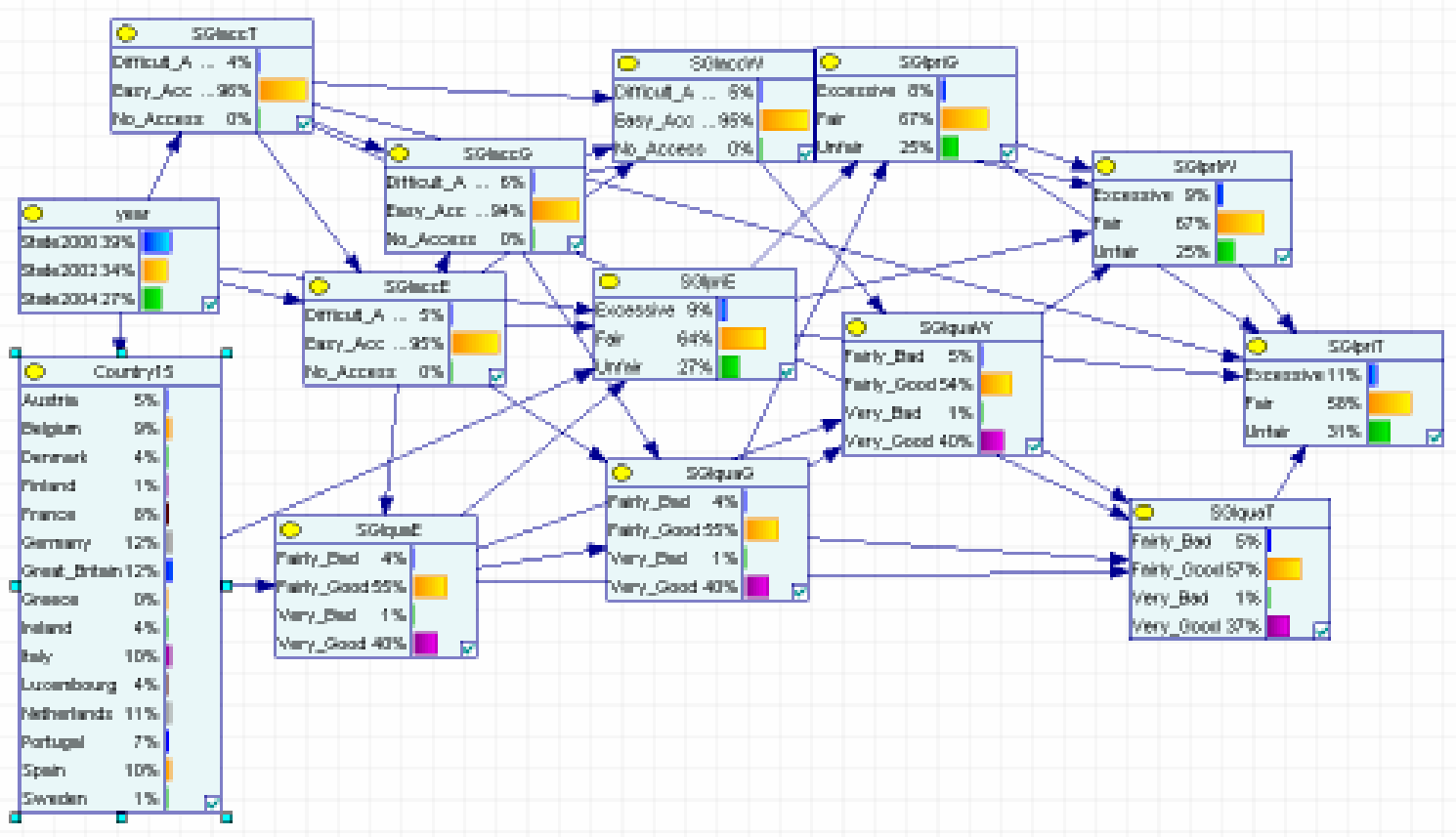

Figure 6: Bar chart Bayesian Network on Eurobarometer Survey data

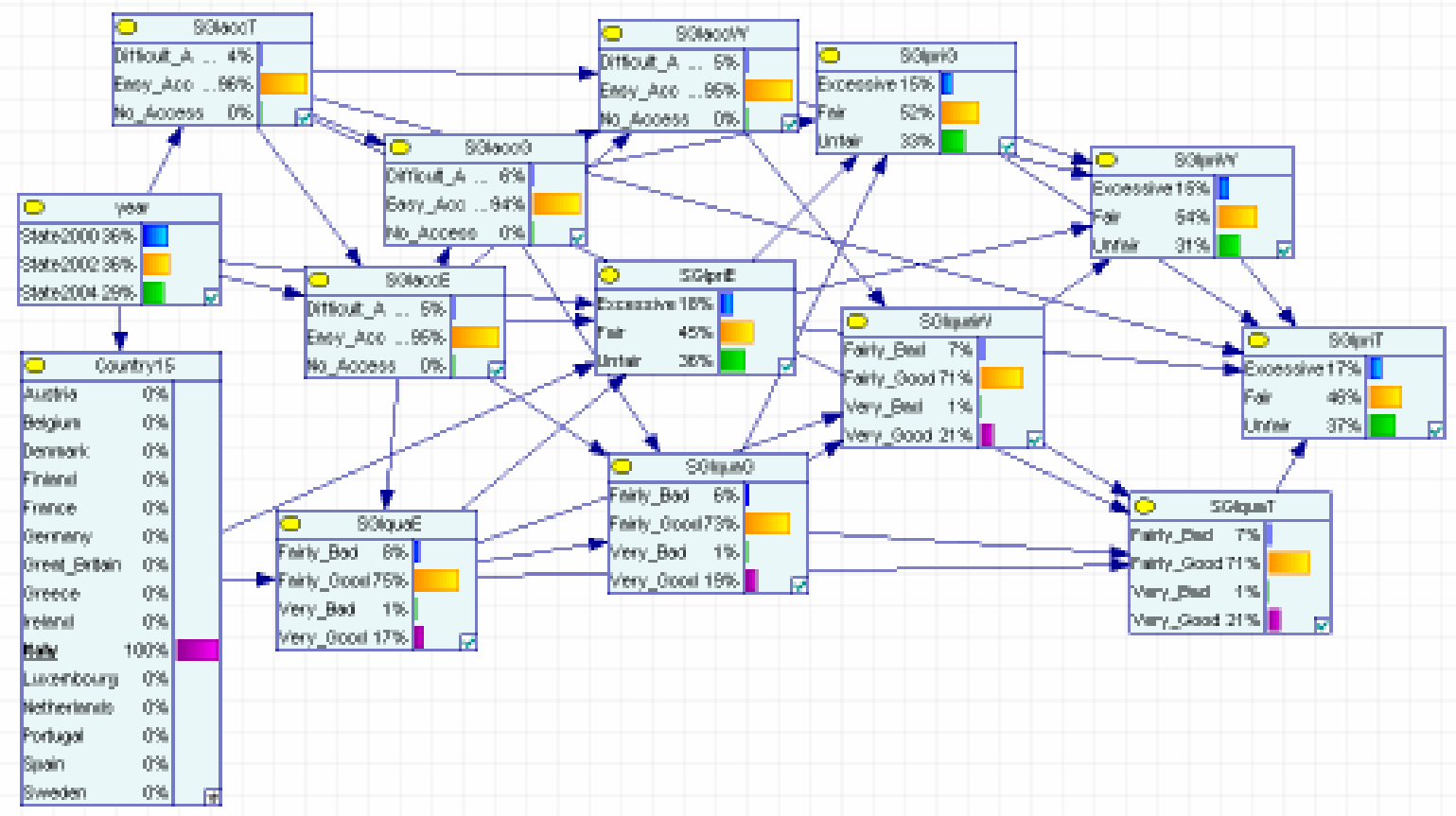

Figure 7: Evidence for Italy 


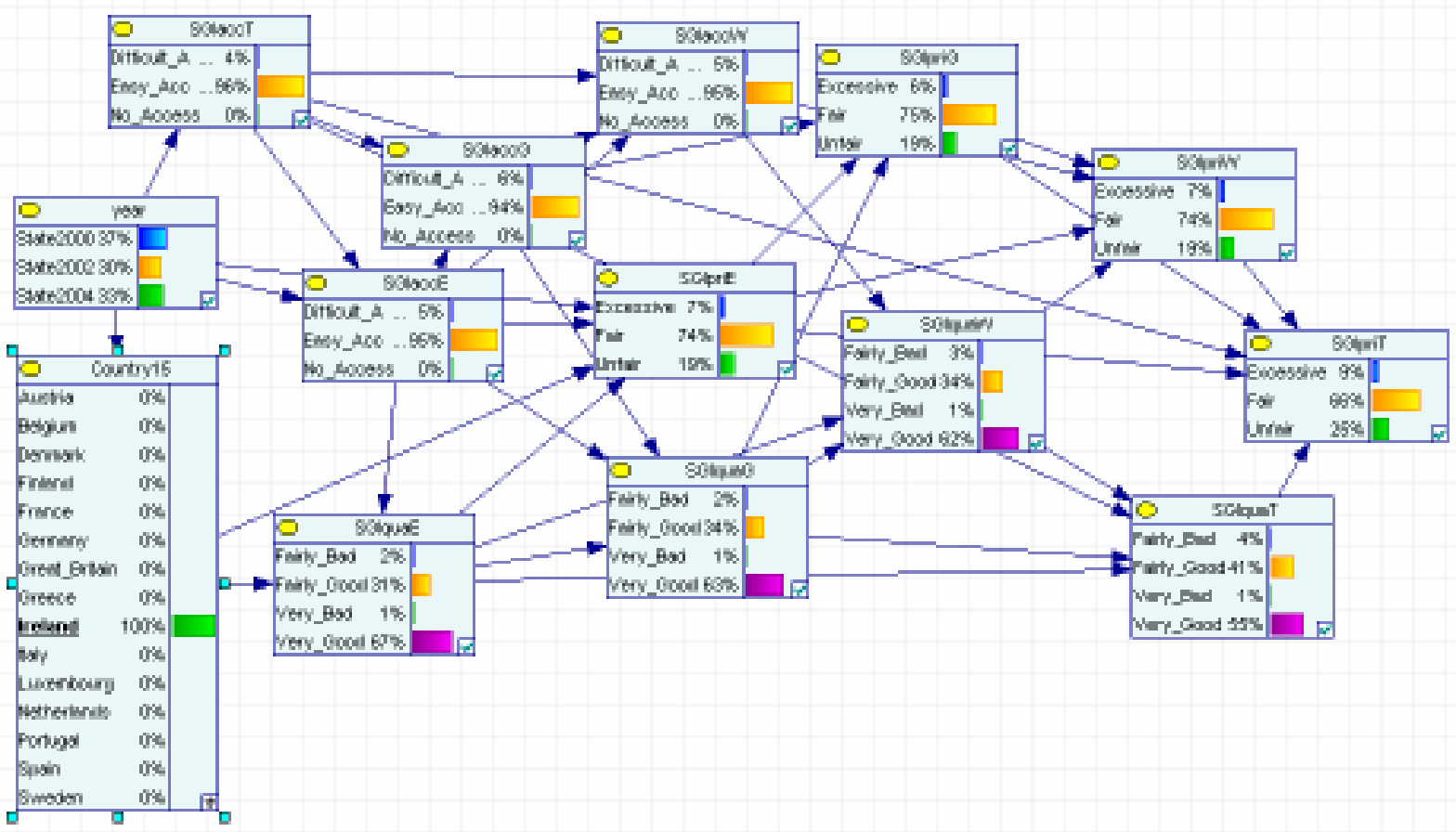

Figure 8: Evidence for Ireland

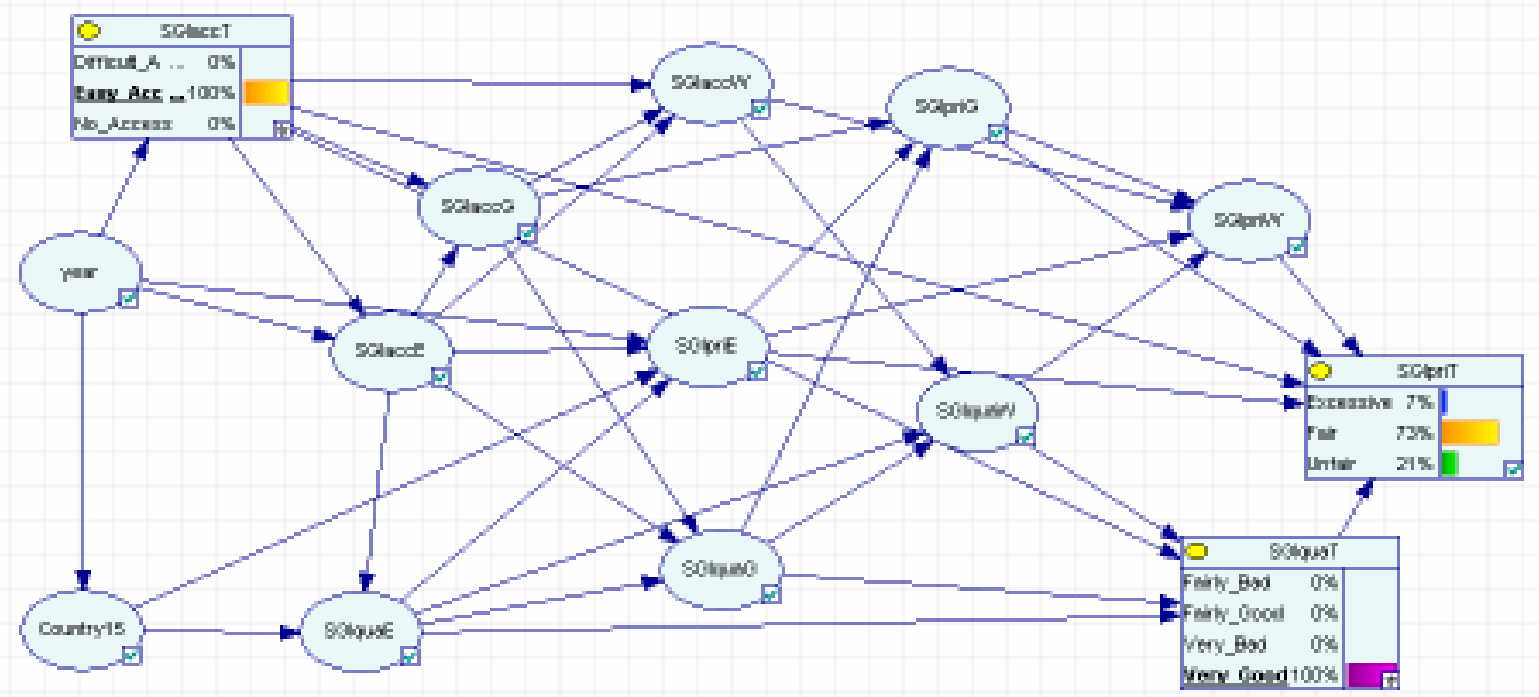

Figure 9: Diagnostic distributions of price of fixed telephone service conditioned on the access of fixed telephone service and quality of fixed telephone service being at their highest level. 


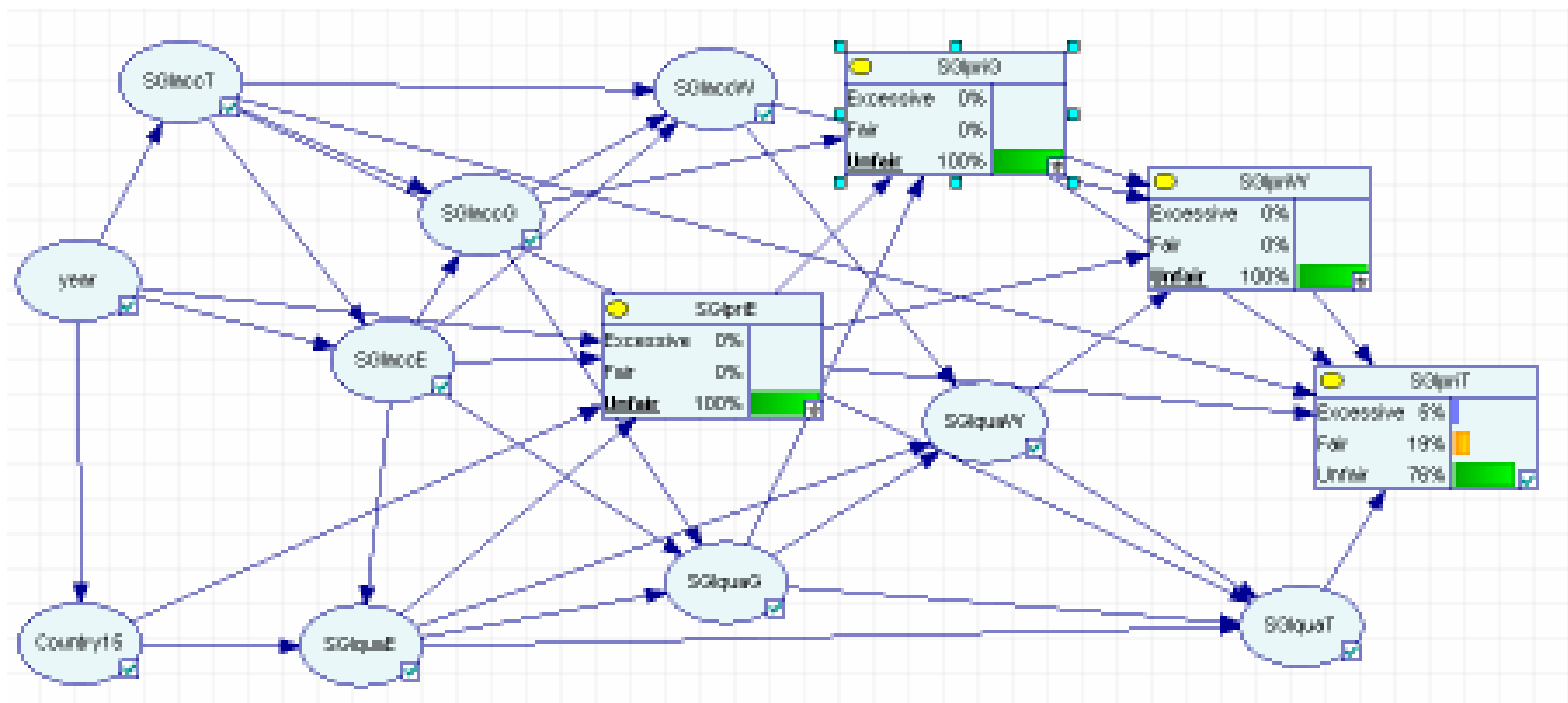

Figure 10: Diagnostic distributions of price of fixed telephone service conditioned on the price of electricity supply service, price of water supply service and price of gas supply service being at their lowest level 
Silvia Salini holds a degree in Statistics from Catholic University of Milan and Phd in Statistics from University of Milan Bicocca. Currently she is Assistant Professor of Statistics at the Department of Economics, Business and Statistics of the University of Milan. Her main research interests are multivariate statistical analysis, data mining and statistical for social science.

Department of Economics, Business and Statistics,

University of Milan. Via Conservatorio 7, 20122 Milan (Italy)

Email: silvia.salini@unimi.it

Tel. $+39(0) 250321538$

Fax $+39(0) 250321450$

Ron S. Kenett is CEO of KPA Ltd., a management consulting firm with offices in Raanana, Israel and Professor at the University of Turin, Turin, Italy. He has written 4 books on applications of Statistics, is an Editor in Chief of the Wiley Encyclopaedia of Statistics in Quality and Reliability and published over 130 papers in scientific journals. His main research interests are multivariate analysis, industrial statistics, strategic analysis models, change management and risk management. Ron is past president of ENBIS, the European Network of Business and Industrial Statistics and Fellow of the Royal Statistical Society.

KPA Ltd., P.O.Box 2525,

Raanana 43100, ISRAEL

Email: ron@kpa.co.il

Phone:+972(0)97408442

Fax: +972(0)97408443 